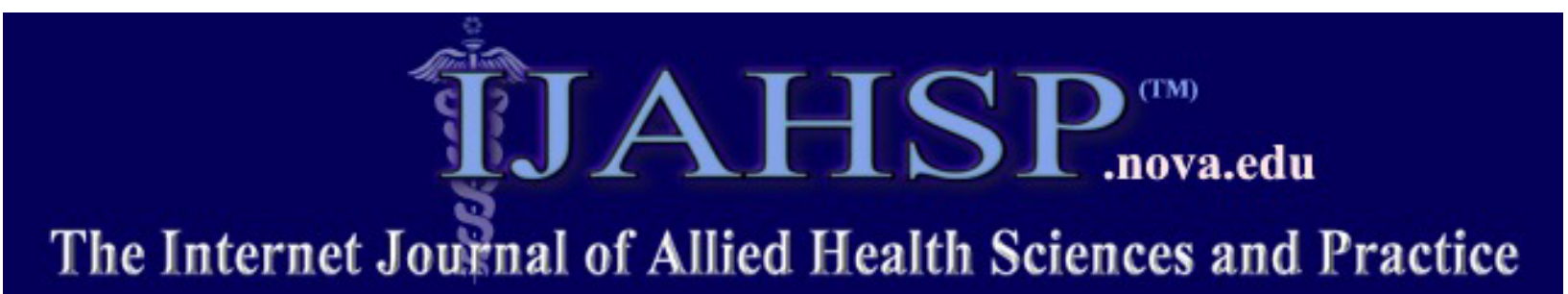

A Peer Reviewed Publication of the College of Health Care Sciences at Nova Southeastern University

Dedicated to allied health professional practice and education

http://ijahsp.nova.edu Vol. 10 No. 3 ISSN 1540-580X

\title{
The Implementation of Evidence to Improve Falls Prevention: Lessons from a Case Study in Aged Care
}

\author{
Gisela M. van Kessel, MHSM, DrPH Candidate \\ Lecturer, Centre for Allied Health Evidence, University of South Australia, Adelaide, South Australia
}

Australia

CITATION: van Kessel GM. The Implementation of Evidence to Improve Falls Prevention: Lessons from a Case Study in Aged Care. The Internet Journal of Allied Health Sciences and Practice. July 2012. Volume 10 Number 3.

\begin{abstract}
Purpose: This paper reports on a study investigating the construction of falls prevention evidence within an aged care organization. It describes how the structure and culture of an aged care organization influences the construction of falls prevention evidence by staff. Method: An instrumental single case study design grounded in an interpretive, constructivist paradigm drew on data collected in 2010 from a document review, observations, and informal interviews with nursing and allied health staff. An iterative analysis used a model of diffusion determinants. Results: The aged care organization which served as the case for this study linked its cultural strengths of vision, leadership, and innovativeness with its decision making structures to resources from its external environment to implement falls prevention strategies that were both best practice and innovative. The case study demonstrated that in addition to known individual and organizational barriers to the use of evidence, the nature of evidence itself can act as a barrier when it is constructed into a norm which directs practice in a particular direction thus obscuring alternatives. Conclusion: Organizations can reflect on how their culture and structure can influence the effective use of evidence, the contribution to creating a social norm that inhibits the creative research, and the management required to address complex health issues.
\end{abstract}

\section{INTRODUCTION}

Falls in older people are a significant public health issue. Globally, fall related injuries are related to substantial economic costs which are predicted to rise with increases in the aged population. ${ }^{1-3}$ In Australia, falls mortality accounts for $62 \%$ of all injury deaths for people over 75 years of age and falls morbidity includes fractures and head injuries with up to $80 \%$ resulting in permanent disability. 4,5 Other impacts on public health arise from the secondary consequence of falls such as a restriction of activity levels and subsequent impaired social interaction. ${ }^{6}$

Approximately $70 \%$ of falls are a result of multiple interacting risk factors that have been summarized from numerous epidemiological studies into guidelines.7,8 Previous systematic reviews have synthesized the substantial body of evidence demonstrating the effectiveness of multi-factorial interventions. ${ }^{9,10}$ These reviews contribute to the risk discourse rather than taking a population-based approach. ${ }^{9}$ Furthermore, implementing falls prevention evidence is not straightforward, with a large section of literature devoted to investigating the facilitators and barriers, at both individual and organizational levels. 13-16,19,20,22,23

Evidence can be defined as "knowledge derived from a variety of sources that has been subjected to testing and has been found to be credible."11 The term "evidence" in this paper refers to knowledge derived from research. ${ }^{12}$ Research-based knowledge is new information that an organization can utilize and as such is an innovation. Evidence is often positioned as a neutral entity but can be conceived as something that has different socially constructed meanings. Using evidence can be considered a process for decision-making. ${ }^{12}$ Much of the literature on the use of evidence investigates how individuals utilize evidence in their decision- 
making. Professionals report that the barriers they face in adapting and reconstructing evidence for different client groups include access, time, critical appraisal skills, knowledge of statistics, and authority to change practice. ${ }^{13-16}$

In addition to these barriers experienced by individuals, the use of evidence also depends in part on the support of the organization and its environment, and these may be more predictive of evidence use than individual factors. ${ }^{13,17-19}$ The internal context of the organization is a complex interaction of social forces shaped by its structure and culture. ${ }^{20}$ The external context influences the use of evidence through inter-organizational networks, uncertainty or competitiveness in the environment, and political imperatives. ${ }^{21}$

Organizational facilitators include leadership, stakeholder support, commitment to education, effective team work, successful past organizational change, and a positive work environment, while barriers include work force instability, negative experiences with organizational change, and uncertainty of the future. $19,20,22,23$

It is recognized that enhancing the use of evidence is not as simple as addressing single variables such as training staff or providing support to a team. There is no clear evidence that changing infrastructure enhances the use of evidence. ${ }^{24}$ Facilitators and barriers occur at interrelated levels such as the client interface, the team, the organization, and the health system. ${ }^{19}$ These require a complex response by staff utilizing skills in organizing and maintaining links across teams and organizational boundaries. ${ }^{19}$

To assist organizations to fully utilize the depth of knowledge available on falls prevention and realize improved public health outcomes, further research is required to understand the mechanisms of the links between the variables as they occur in the real world context. ${ }^{21}$ This study was designed to describe the use of evidence for falls prevention by an organization in order to contribute to an understanding on how to optimize public health practice. The aims of the study were to: i) to describe how the organizational structure influences the construction and use of evidence in the practice of falls prevention, ii) and to explore the influence of organizational culture on that use.

\section{METHOD \\ Design}

An instrumental single case study design, grounded in an interpretive, constructivist paradigm was used.25 The case study method allowed an in-depth investigation of a contemporary phenomenon, where sources of knowledge and behaviors were not manipulated. ${ }^{25-28}$ The use of an instrumental case study enabled the use of falls prevention evidence within an organization as the principle research focus rather than an interest in a particular case. ${ }^{25}$ The choice of a single case provided an opportunity for learning unfettered by competing interests and enabled a rich description to be developed. 25,26 An interpretive, constructivist analysis was particularly suited to the study aim, exploring the construction of knowledge within an organization.

\section{Sample}

The organization used in this case study was purposively chosen on the basis of the opportunities it offered to learn rather than its ability to be representative. ${ }^{25} \mathrm{It}$ is a complex and large not-for-profit aged care organization based in a metropolitan city in Australia. The organization provides a range of housing which includes units where residents are relatively independent and nursing home accommodation. In addition, the organization offers a range of services to older people in the community including therapy, respite, and assistance with domestic, personal, and nursing care in the home, and short term transition services. It employs a range of managers, professionals, care and support staff. Participants were recruited through a manager who located staff that were willing to be informants and act as a guide to the organization's cultural mores. ${ }^{29}$ Participants were recruited on the basis of their interest and involvement within the organization with falls prevention activities. Eight participants from management, nursing, and therapist backgrounds assisted the researcher to expand her knowledge of the structural influences and develop an understanding of the cultural influences on the use of evidence in the case.

\section{Data Collection}

The case study was conducted during a four month period in 2010. A document analysis of organizational charts, annual report, terms of reference, and falls screening and assessment forms was the initial method used to gain an understanding of the organizational structures that supported or undermined the use of evidence in the practice of falls prevention. Further data on culture and structure was collected from participants by observing of the Falls Prevention Working Group. Four of the participants also took the researcher on tours with interviews through the workplace. The researcher presented herself as a learner rather than an expert to gain trust and establish a rapport through open ended questions..$^{29}$ The interviews were digitally recorded and transcribed verbatim by the researcher. The participants checked the transcripts and gave permission for quotes to be used. 


\section{Ethical Considerations}

This case study received ethical approval from a university research ethics committee. Participants received a letter of introduction and an invitation to participate, and completed a written consent form prior to participation.

\section{Data Analysis}

An inductive and iterative data analysis was conducted using the model, Determinants of diffusion of innovations in the organization and delivery of health service developed by Greenhalgh and colleagues to understand the diffusion of innovations in health care organizations. ${ }^{21}$ The model is based on a meta-narrative review of the literature, using an iterative and pluralist approach, and as such, enables triangulation of theory. The model is underpinned by 13 research traditions including particularly relevant theories on health promotion, evidence-based medicine, and organizational structure and culture. The model describes the inner context of the organization that includes the constructs of system antecedents, system readiness, adoption/assimilation, implementation within the system, and consequences. The outer context is considered to include the constructs of resource system, knowledge purveyors, and change agency. The linkages between the inner and outer context play a central role in the model. The analysis was conducted by interrogating all sources of the data using each component of the model. This analysis was followed by an interpretation of the complex interaction and linkages between the components as they relate to this specific case study.

\section{Rigour}

The multiple sources of data were managed in a database to maintain a chain of evidence to achieve construct validity and triangulation. ${ }^{27,28,30} \mathrm{~A}$ review of the literature was used to guide the initial document review, which in turn provided background context to the interviews. The process of participants leading the researcher on a tour allowed the participant to control the information and minimise the persona of the researcher as a person who privileges research knowledge. The interview transcripts were checked for accuracy by sending them to the participants.

\section{RESULTS}

The organization has a matrix structure consisting of divisions and professional groups. The division structure was the stronger framework and both facilitated and inhibited the use of evidence. It enabled effective downward dissemination of knowledge but limited upward flow of feedback. Information flow across divisions was formally supported by the Clinical Governance Committee and Falls Prevention Working Group.

Much of the organization's success may be attributed to a culture that supported the use of evidence through proactive leadership, a cohesive management team, a strategic vision that focused around positive ageing, and a climate that supported experimentation. Innovative ideas were supported by providing significant resources to undertake activities such as international study tours and extensive research of the literature.

Figure 1. Determinants of Diffusion of Falls Prevention Evidence in an Aged Care Organization

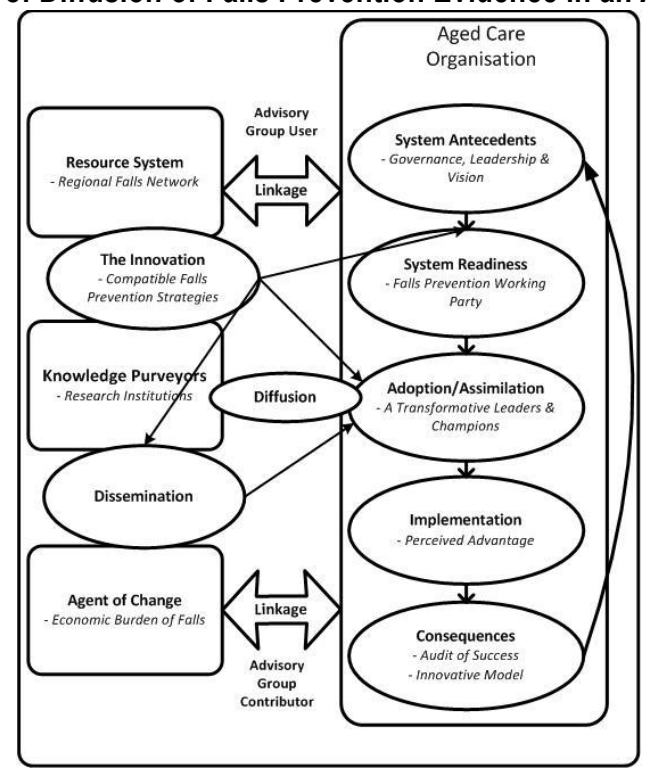

*Adapted from Greenhalgh et al 2005, p. 8 
Outer Context and Linkages - Inter-Organizational Networks and Collaboration

The key resource systems that provided falls prevention evidence for the organization identified by all participants were the two regional falls networks. The agent of change that led to the development of the networks was the political concerns regarding the economic burden of falls. These concerns were acted on through the establishment of action research projects culminating in setting up two local networks. The networks provided evidence synthesized into a range of products. These included locally developed tools such as brochures, posters, screening and assessment forms, as well as distributing nationally developed resources such as guidelines and resource kits.

Staff have developed ongoing links with the local regional network at the management level, participating in the advisory group, while clinicians made substantial use of the training opportunities offered. Thus the organization both contributed to, and utilized the services of the networks.

'We made a point to be linked into all the regional falls prevention work to learn as an organization. I am on an advisory group for Falls Prevention with State Health. So forming those links and always checking where we are in comparison to new information coming in helps us.' (Manager A)

\section{Inner Context - System Antecedents/Readiness}

The Clinical Governance Committee was a key structural influence on the use of evidence. Managers felt it supported them to maintain links across teams and organizational boundaries, helping to overcome the silos created by the division structure. A matrix structure was also used by management at the next level down to support professional staff. It allowed professionals to work together in streams which cut across the division structure and enhanced the capacity for the use of evidence.

It was the first time the organization had tried to implement something [Falls Prevention Working group] organizationally wide, where we are quite a siloed organization, so we set up an interdivision group that had representation from senior management, from clinical, nursing, allied health and our team coordinators.' (Manager A)

The dissemination of evidence from the leadership group and within the organization level was thought to be well supported by the structure by both managers and professionals, but the flow upwards was felt by therapists in particular to be hampered by the level of authority an individual staff member had. While there was a process for employees to raise concerns and ideas, there was no evidence that this was systematically embedded in the structure.

'I think if I wasn't part of the [Falls Prevention] working groups I would find it hard. I don't know if other people in the organization would be exploring for a while trying to figure who is the person to ask. I think in these big organizations having your pulse on what is happening can be quite difficult.' (Therapist)

\section{Adoption/Assimilation}

Managers and professionals identified that the products provided through the networks enhanced the success of some areas of falls prevention evidence. The networks were identified as playing a significant role in reducing the complexity that the large volume and often contradictory or incomplete evidence can create. The products were seen as compatible with the organization's values around positive ageing and as such were adopted without much question by staff. The screening and assessment tools were seen by both therapists and nurses as easy to adapt to the organization's needs and required minimal management time to develop.

'There is no room for error with the [falls] screening tool because it is very simple and it is very, you know, the parameters are very neat, so it is really what to do with the screening tool, where we can work on it and improve it like add a flow chart or some tick boxes.' (Nurse)

The assimilation of falls prevention evidence by the organization was an iterative, rather than linear or stage process. Managers had a positive influence as opinion leaders and acted as champions at an organizational level. These managers have used a transformational leadership approach, drawing on their external networks and utilizing internal relationships across divisions, to introduce a number of innovations. New ideas were brought to the Clinical Governance Committee where managers felt there was a culture of supporting creativity. Innovations were reported as having been adopted and assimilated although some also languished for a while when other priorities needed to be addressed, but were then revived and supported. The Clinical Governance Committee used formal and centralized structures such as the Falls Prevention Working Group to involve clinicians in management networks in a vertical structure, rather than being diffused through informal decentralized professional networks. 
'One of the things with this organization is that really anyone in the organization that has a passion or a vision around an important issue usually can find a mechanism for bringing that up.......so we used the forum of senior managers to get endorsement for that [falls prevention] and we were really encouraged.' (Manager A)

\section{Implementation}

Consistent implementation was the primary concern for managers. The screening tool was reported to have been adopted more effectively than the falls risk assessment or data collection process. This was explained by a perceived advantage of the screening tool, in terms of its simplicity and ease of use, as well as its observable categorization of clients. In contrast, managers felt the advantage of data collection was not be immediately obvious to professionals due to time lags in results, and the effects of assessment dependent on client uptake of the evidence-based advice. The assessment tool was not perceived by staff in particular as providing all the information they needed to convince clients. It reflected the narrow injury prevention perspective of much of falls prevention evidence and did not address a wellbeing approach to promoting mobility.

'So sometimes the client is screened as high risk and nothing happens because the coordinators will say "I can refer you to a falls prevention groups at this day therapy centre or I can get the physiotherapist to see you at home" and the client declines.' (Nurse)

Despite these limitations, the uptake of evidence by professionals was strong, as evidenced by an audit of screening completions. The motivation of professionals appeared to be driven by the powerful influence of organizational values based on creating good lives for older people. This was further reinforced through strong organizational support to attend workshops which increased the capacity of individuals to take up falls prevention evidence. However there was less evidence that professionals sought out the literature themselves.

'There is definitely support from our team leaders and manager to be part of the Falls group and work on it. In terms of finding time day to day to do some research, I guess that does get tricky, it gets to the bottom of the pile.' (Therapist)

Evidence on the effectiveness of champions has led to the use of Falls Champions as a strategy to further implement and institutionalize falls prevention. The organization has failed to set up a sustainable system, primarily as a consequence of staff turnover. The strategy was being revisited and built into the structure more systematically through job descriptions and by appointing care workers to the role of champions. The experience has been that these workers have more capacity in terms of time and motivation through increased diversity of their role and job satisfaction.

'Generally when we pick care workers as Falls Champions it increases their job satisfaction and they are actually really good champions and they take it really seriously. It is probably their only other portfolio that they have from their day to day work.......so they are really good at spreading it and making people remember it and see the importance of it.' (Nurse)

\section{Consequences - Falls Prevention in an Active Ageing Framework}

The organization's culture enabled it to look beyond the risk discourse that is the social norm and use evidence to develop their active ageing model of care. Their model is drawn from evidence that directs a focus on supporting mobility and participation as much as it does on keeping residents safe. Resources were allocated to a new facility where evidence was used to create a physical and social environment that supports mobility. The model is based on evidence from the positive ageing, active ageing, and positive psychology disciplines that are consistent with the organization's values.

'The model is about 7 key understandings that relate to a good life. It is all based on research. There are three inter disciplines that we drew from which is positive ageing, active ageing, and positive psychology. It is a contemporary model. It's based on what we would expect for a life course so it is not just fitting for aged care, it is fitting for all staff.' (Manager B)

The concept of champions was used again to reinforce a new social environment. The design of the physical environment used evidence to inform decisions such as floor coverings and architecture. The result was an environment that encouraged mobility with inviting indoor and outdoor spaces such as courtyards, balconies, vistas and large open doors. It promoted activity with areas such as the gym, church, cafe, and cultural meeting room which supplemented the more commonly seen communal living and dining areas. The physical environment was extended beyond the building through the use of a bus which took residents to swimming sessions, shopping, mystery tours, and walks in the park.

'Down the stairs there will be a mini golf range. Everything is about activity, going out in the bus, they go to an activity. There is going to be a gardening shed developed up there somewhere. The stairs are going to be turned into the Stairs of Strength, 
where you can go and do a work out. There is a walking trail that is developed and that we'll have the trail put up here and residents can put in how many kilometers they are walking and our goal is to get to Mt Everest and things like that. The champions are running these projects, they all get 500 dollars to run a project and to roll it out, but it is all about keeping mobile and stopping falls. Ultimately that is the key.' (Manager B)

\section{DISCUSSION}

The observation of an organization utilizing the model of Determinants of diffusion of innovations in the organization and delivery of health service developed by Greenhalgh and colleagues has identified how this case implemented falls prevention strategies that were both best practice and innovative. ${ }^{21}$ Falls prevention practice was supported by effective links between the organization's cultural strengths of vision, leadership, and innovativeness with its decision making structures to resources from its external environment.

This real life case demonstrates the structural barriers and facilitators previously identified in the literature and provides a deeper understanding of the important links between the internal and external networks for the use of falls prevention evidence. 13-20,22,23 The key linkages this organization used included the participation through external training opportunities and contributions through advisory group membership, which ensured successful collaboration with inter-organizational networks. This was optimized by the skills of the leadership team in organizing, facilitating, and maintaining links across the organizational boundaries, and effectively resourcing innovation. The greatest threat to effective falls prevention practice came from inside the organization due to workforce instability and turnover which result in a loss of intellectual capital and increased training costs. One consequence was that momentum gained by an initiative such as the Falls Champions could then stall or be lost altogether.

The culture of the organization has what Greenhalgh and colleagues call "absorptive capacity for new knowledge". ${ }^{21}$ As an organization it has been successful in its use of evidence to contribute to public health practice through its capacity to look beyond the dominant risk discourse, to evidence from a wellbeing perspective. Managers have used their passion, vision, and existing knowledge and skills to reconstruct evidence and reframe injury prevention as enabling mobility through a social participation and empowerment model. Managers were supported by the organization which has invested significantly in developing a new culture and physical environment to take a community approach to falls prevention that encompassed staff, older people, and their families.

This contrasts to the normative risk approach and highlights how keeping to the main body of evidence may constrain the development of public health practice. As professionals and organizations collaborate to adapt complex evidence to suit their particular environment, they also socially construct its meaning. When this is particularly successful, the shared meaning develops a norm, which in turn facilitates dissemination. The norm of keeping older people safe and preventing injury has contributed to successful falls prevention evidence dissemination but also obscured a wellbeing approach that enables activity.

\section{Limitations}

This case study allowed the researcher to develop an in depth understanding of the real life use of falls prevention evidence but there may have been alternative views held by staff that did not participate in the study that were not able to be explored. The four month duration of the case study has meant that only the concerns confronting the organization at a particular point in time have been observed. An example of this is the Falls Prevention Working Group focus on the process of reviewing assessment procedures. The plan was to move towards developing interventions within the community. The description and subsequent analysis in this case study may be biased towards how the organization used evidence in assessment of risk. Had the case study occurred when interventions were being developed this may have been less apparent.

\section{Implications}

Despite these limitations which reduce the generalizability and transferability of the results, this case study demonstrates how managers can use the model developed by Greenhalgh and colleagues to assess the barriers to the use of evidence in their own organization. ${ }^{21}$ Understanding that evidence is constructed and used in a non-linear process that involves a dynamic interaction between structure and culture, both within and external to their organization, may help managers to enhance their capacity for effective public health practice.

Researchers can position themselves within networks to enhance the dissemination of their work. With an understanding that some of the problems of dissemination lie in the evidence itself, they can reflect on the need to increase the usability of their findings by decreasing complexity, improving trialability, or promoting the relative advantage of their findings. While building evidence on knowledge obtained from previous work is essential, research questions should also challenge assumptions and norms. 


\section{CONCLUSION}

This case study observed the real life occurrence of structural facilitators and barriers to the use of evidence at both individual and organizational levels found previously in the literature. However barriers also existed in the nature of the evidence itself. These included its complexity and the way evidence is constructed into a norm which can constrain public health practice. The case study has described how an organization that operated in the dominant risk paradigm, but utilized its inner strengths of leadership and vision, can develop a culture that uses evidence in an innovative way to create a new model of care which focuses on wellbeing to achieve public health outcomes.

\section{REFERENCES}

1. Moller J. Projected costs of fall related injury to older persons due to demographic change in Australia. Commonwealth Department of Health and Aging; Canberra. 2003.

2. Iglesias C, Manca A, Torgerson D. The health related quality of life and cost implications of falls in elderly women. Osteoporos Int. 2009; 20:869-78.

3. Bohl A, Fishman P, Ciol M, Williams B, LoGerfo J, Phelan E. A longitudinal analysis of total 3-year healthcosts for older adults who expereince a fall requiring medical care. J Am Geriatr Soc. 2010; 58(5):853-60.

4. Braithwaite R, Col N, Wong J. Estimating hip fractures morbidity and mortality and costs. J Am Geriatr Soc. 2003; 51:36470.

5. Pointer S, Harrison J, Bradley C. National Injury Prevention Plan priorities for 2004 and beyond: Discussion paper. Australian Institute of Health and Welfare; Canberra. 2003.

6. Vellas B, Wayne S, Romero L, Baumgartner R, Garry, P. Fear of falling and restriction of mobility in elderly fallers. Age Ageing.1997; 26(3):189-93.

7. American Geriatrics Society, British Geriatrics Society and American Academy of Orthopedic Surgeons Panel on Falls Prevention. Guideline for the prevention of falls in older persons. J Am Geriatr Soc. 2001; 49(5):664-72.

8. Campbell A, Robertson M. Implementation of multifactorial interventions for fall and fracture prevention. Age Ageing. 2006; 35 (Suppl ii):60-4.

9. McClure R, Turner C, Peel N, Spinks A, Eakin E, Hughes K. Population-based interventions for the prevention of fall-related injuries in older people (Review). Cochrane Databases Syst Rev. 2005; 1.

10. Gillespie L, Robertson M, Gillespie W, Lamb S, Gates S, Cumming R, Rowe B. Interventions for preventing falls in older people living in the community. Cochrane Databases Syst Rev. 2009; 2.

11. Jones $\mathrm{M}$, Higgs J. Will evidence -based practice take the reasoning out of practice? In: Higgs J, Jones M, editors. Clinical reasoning in the health professionals, 2nd ed. Boston: Butterworth Heinemann; 2000. p. 307-15.

12. Scott-Findlay S, Pollock C. Evidence, research, knowledge: A call for conceptual clarity. Worldviews Evid Based Nurs. 2004; 1:92-7.

13. Estabrooks C, Floyd J, Scott-Findlay S, O'Leary K, Gushta M. Individual determinants of research utilisation: A systematic review. J Adv Nurs. 2003; 43:506-20.

14. Bryar R, Closs S, Baum G, Cooke J, Griffiths J, Hostick T, Kelly S, Knight S, Marshall K, Thompson D. The Yorkshire BARRIERS project: diagnostic analysis of barriers to research utilisation. Int J Nurs Stud. 2003; 40:73-84.

15. Iles R, Davidson M. Evidence based practice: a survey of physiotherapist's current practice. Physiother Res Int. 2006; 11:93-103.

16. Schoonover H. Barriers to research ultization among registered nurses practicing in a community hospital. J Nurses Staff Dev. 2009; 25:199-212.

17. Rubin G, Frommer M, Vincent N, Phillips P, Leeder S. Getting new evidence into medicine. Med J Aust. 2000; 172:180-3.

18. Cockburn J. Adoption of evidence into practice: Can change be sustainable? Med J Aust. 2004; 180:S66-7.

19. French B. Contextual factors influencing research use in nursing. Worldviews Evid Based Nurs. 2005; 2(4):172-83.

20. Scott S, Estabrooks C, Allen M, Pollock C. A context of uncertainty: How context shapes Nurse's research utilisation behaviours. Qual Health Res. 2008; 18:347-57.

21. Greenhalgh T, Robert G, Bate P, Macfarlane F, Kyriakidou O. Diffusion of innovations in health service organisations: a systematic literature review. Malden (Mass): BMJ Books/Blackwell Publishing; 2005.

22. Hamilton S, McLaren S, Mulhall A. Assessing organisational readiness for change: use of diagnostic analysis prior to the implementation of a multidisciplinary assessment for acute stroke care. Implement Sci. 2007; 2:21.

23. Nilsson-Kajermo K, Undén M, Eriksson L, Orton M, Arnetz B, Nordstrom G. Predictors of nurses' perceptions of barriers to research utilisation. J Nurs Manag. 2008; 16:305-14.

24. Foxcroft D, Cole N. Organisational infrastructures to promote evidence based nursing practice Cochrane Databases Syst Rev. 2000; 3.

25. Stake R. Case studies In: Denzin NK, Lincoln YS, editors. Handbook of qualitative research. 2nd ed. Thousand Oaks: Sage Publications, 2000. p.435-54. 
26. Darke P, Shanks G, Broadbent M. Successfully completing case study research: combining rigour, relevance and pragmatism. Information Systsems Journal.1998; 8:273-89.

27. Yin R. Case study research design and methods. Thousand Oaks: Sage Publications; 2003.

28. Anthony S, Jack S. Qualitative case study methodology in nursing research: an integrative review. J Adv Nurs. 2009; 65:1171-81.

29. Fontana A, Frey J. The interview: From structured questions to negotiated text. In: Denzin NK, Lincoln YS, editors. Handbook of qualitative research. 2nd ed. Thousand Oaks: Sage Publications; 2000. p.645-72.

30. Farmer $\mathrm{T}$, Robinson $\mathrm{K}$, Elliott $\mathrm{S}$, Eyles $\mathrm{J}$. Developing and implementing a triangulation protocol for qualitative health research. Qual Health Res. 2006; 16:377-94.

\section{KEY TERMS}

Evidence-based Innovation, Research Utilisation, Diffusion, Organizational Context, Falls Prevention 